\title{
Multipair two-way massive MIMO AF relaying with ZFR/ZFT and hardware impairments over high-altitude platforms
}

\author{
SUN Xiaoli ${ }^{1}$, XU Kui ${ }^{1}$, XU Youyun ${ }^{1,2}$, MA Wenfeng ${ }^{1}$ \\ 1. PLA University of Science and Technology, Nanjing 210007, China \\ 2. Nanjing University of Posts and Telecommunications, Nanjing 210023, China
}

\begin{abstract}
HAPs (High-Altitude Platforms) are one of the most promising alternative infrastructures for providing wireless communications to overcome the shortcomings of terrestrial-tower-based and satellite systems. This paper investigates a multipair two-way MM-AF (Massive MIMO Amplify-and-Forward) relay system over HAPs, where multiple user pairs exchange information within a pair through a relay with a very large number of antennas, and a HAP channel is modeled to follow a Rician fading distribution because of the presence of a line-of-sight path. First, the effect of hardware impairments on a multipair two-way MM-AF system is taken into consideration and is modeled using transmit and receive distortion noises. Then, ZFR/ZFT (Zero-Forcing Reception/Zero-Forcing Transmission) processing matrices of HDR (Half-Duplex Relaying) and FDR (Full-Duplex Relaying) with imperfect channel state information are presented. Finally, the asymptotic expressions of an end-to-end SINR are derived. Theoretical analyses and simulation results show that when the number of relay antennas grows very large, the SE (Spectral Efficiency) of an MM-AF relay system is limited by hardware impairments at the users instead of those at the relay, or by other types of interference. Thus, the quality of the hardware at a relay can be decreased without significantly degrading performance.
\end{abstract}

Key words: massive MIMO, half-duplex, full-duplex, Rician fading channels, hardware impairments

\section{Introduction}

HAPs (High-Altitude Platforms) are novel alternate technologies that provide new possibilities for wireless communications such as large coverage areas, less hardware, high data rates, and short propagation delay while combining these advantages in both terrestrialbased ground and satellite systems. One deployment scenario of MIMO (Multiple-Input Multiple-Output) over HAPs is a flying aircraft (relatively stationary with respect to the ground) carrying a horizontal planar array antenna, providing service to the users

Manuscript received Sept. 8, 2016; accepted Sept. 26, 2016

This work is supported by the Major Research Plan of the National Natural Science Foundation of China (No.91438115), the National Natural Science Foundation of China (Nos.61371123, 61301165, 61671472), Jiangsu Province Natural Science Foundation (No.BK20160079), Special Financial Grant of The China Postdoctoral Science Foundation (No.2015T81079), China Postdoctoral Science Foundation (No.2014M552612), Jiangsu Postdoctoral Science Foundation (No.1401178C), the Preresearch Project Foundation (No.9140C020306130C02007). 
on the ground. The Google Balloon and the "Internet from the Sky" proposed by Facebook are examples of this scenario.

Massive MIMO is a promising technology to meet the ever-increasing data-rate demand in $5 \mathrm{G}$ (fifthgeneration) mobile communications. It is a robust, secure spectrum and is energy efficient ${ }^{[1]}$. It is natural to consider a massive MIMO scheme, i.e., a large antenna array that serves multiple users on the same channel. The challenge nowadays is to investigate the application of Massive MIMO techniques to HAPbased communication systems.

Many existing works considered HDR, where the relay transmits and receives using an orthogonal frequency or time resources ${ }^{[2]}$. FDR has recently attracted considerable attention as an approach to double the SE of traditional $\mathrm{HDR}^{[3]}$. However, the main obstacle faced by FDR is its difficulty in canceling strong EI (Echo Interference) from the relay output to the input ${ }^{[4]}$. A relay system includes one-way and two-way communication ${ }^{[5]}$. A two-way relay system can overcome the inherent time and spectrum resource losses in a one-way relay system. To achieve spectral and energy-efficient transmissions for multiple user pairs, recent works sought to incorporate both $\mathrm{HDR}^{[6,7]}$ and $\mathrm{FDR}^{[8,9]}$ with massive MIMO.

However, most works on massive MIMO have been based on the strong assumption of using perfect hardware in the RF (Radio-Frequency) chains ${ }^{[6-9]}$. In fact, since there are a massive number of antennas, low-cost RF chains are needed to reduce the cost. Lowcost components are prone to hardware imperfections that must be considered in practical systems ${ }^{[10]}$.

To the best of our knowledge, this is the first paper on the study of the effects of residual impairments in multipair two-way massive MM-AF relay systems over Rician fading channels. This is different from works that considered the effects of hardware impairments on single-user and multiuser massive
MIMO base station systems ${ }^{[10,11]}$ and multipair oneway DF-based massive MIMO FDR systems in Rayleigh fading channels ${ }^{[12]}$. Moreover, we consider Rician fading channels, which are the most important models for representing a received signal composed of both diffuse scatter components and a LoS (Line-of-Sight) component, and are typical for HAP communication. In this paper, the effects of hardware impairments on a multipair two-way MMAF system over Rician fading channels is taken into consideration and is modeled using transmit and receive distortion noises ${ }^{[10]}$. The ZFR/ZFT processing matrices of HDR and FDR with imperfect CSI are presented. In addition, the asymptotic expressions of the end-to-end SINR are derived.

Notation: We use $\boldsymbol{X}^{\mathrm{T}}, \boldsymbol{X}^{\mathrm{H}}, \boldsymbol{X}^{*}, \boldsymbol{X}^{-1}$ and $\operatorname{Tr}(\boldsymbol{X})$ to denote the transpose, conjugate-transpose, conjugate, inverse and the trace of $\boldsymbol{X}$, respectively. Moreover, $\boldsymbol{I}_{M}$ denotes an $M \times M$ identity matrix. Finally, $\mathrm{E}\{\bullet\}$ is the expectation operator.

\section{System model}

In a massive MIMO multipair two-way relaying system, $2 K$ users making up $K$ communication pairs, and exchange information within pair by a shared massive antenna AF relay (R). Without loss of generality, a pair of source nodes $\mathrm{U}_{2 l-1}$ and $\mathrm{U}_{2 l}$ are too far apart to communicate directly. Now, we consider two cases in which $\mathrm{R}$ and the users will operate in either HD (Half-Duplex) mode or FD (Full-Duplex) mode.

1) Half-Duplex Relay: In this case, $R$ and the users use two orthogonal time slots. All users have a single antenna, and $\mathrm{R}$ has $\mathrm{M}$ antennas. Additionally, the channel matrix between all the users and $\mathrm{R}$ is denoted by $\boldsymbol{G}_{1} \in \mathbb{C}^{M \times 2 K}$.

Data transmission consists of two phases. During the first phase, all users simultaneously transmit their signals to $\mathrm{R}$. Therefore, the received signal at $\mathrm{R}$ is 


$$
\tilde{y}_{R}=y_{R}+r_{R}=G_{1}\left(x_{s}+t_{R}\right)+n_{R}+r_{R},
$$

where $x_{s}=\left[x_{1}, x_{2}, \cdots, x_{2 K}\right]^{\mathrm{T}}$, and $\mathbb{E}\left\{x_{s} x_{s}^{\mathrm{H}}\right\}=\operatorname{diag}$ $\left(P_{s, 1}, \cdots, P_{s, 2 K}\right)=\Lambda$. The distortion noises are $t_{s} \sim \mathcal{C N}(0$, $\left.\operatorname{diag}\left(v_{1} \mathrm{E}\left[\left|x_{1}\right|^{2}\right], \cdots, v_{2 K} \mathrm{E}\left[\left|x_{2 K}\right|^{2}\right]\right)\right)$, and $r_{R} \sim \mathcal{C N}\left(0, \mu_{R}\right.$ $\left.\operatorname{diag}\left(\mathrm{E}\left[y_{R} y_{R}{ }^{\mathrm{H}}\right]\right)\right)$. Note that $v_{i}=0, i=1, \cdots, 2 K\left(\mu_{R}=0\right)$ corresponds to the conventional assumption of a perfect transmision of RF chains at the users (perfect receive $\mathrm{RF}$ chains at $\mathrm{R}$ ). The quality of the transmit and receive $\mathrm{RF}$ chains degrades as $v_{i}\left(\mu_{R}\right)$ increases. Moreover, $\boldsymbol{n}_{R}$ represents the noise vector at $\mathrm{R}$. The elements of $\boldsymbol{n}_{R}$ are assumed to be i.i.d. $\mathcal{C N}(0, \sigma)$.

During the second phase, $\mathrm{R}$ amplifies the received signal. Then, $\mathrm{R}$ forwards the amplified signal back to the users, and the received signal at the $k$ th user is given by

$$
\tilde{y}_{k}=y_{k}+r_{k}=\boldsymbol{g}_{1, k}^{\mathrm{T}}\left(\boldsymbol{x}_{R}+\boldsymbol{t}_{R}\right)+\boldsymbol{n}_{k}+r_{k},
$$

where the distortion noises $t_{R} \sim \mathcal{C N}\left(0, v_{R} \operatorname{diag}\left(\mathrm{E}\left[x_{R} x_{R}{ }^{\mathrm{H}}\right]\right)\right)$, and $r_{k} \sim \mathcal{C N}\left(0, \mu_{k} \mathrm{E}\left[y_{k} y_{k}{ }_{k}^{\mathrm{H}}\right]\right), k=1, \cdots, 2 K$. Note that $v_{R}=0$ $\left(\mu_{k}=0\right)$ corresponds to the conventional assumption of perfect transmit RF chains at $\mathrm{R}$ (perfect receive $\mathrm{RF}$ chains at the $k$ th user). The quality of the transmit and receive RF chains degrades as $v_{R}\left(\mu_{k}\right)$ increases. $\boldsymbol{x}_{R}$ denotes the transmit vector of R with power $P_{R}=T_{r}$ $\left(\mathrm{E}\left\{\boldsymbol{x}_{R} \boldsymbol{x}_{R}{ }^{\mathrm{H}}\right\}\right)$. Moreover, $\boldsymbol{n}_{k}$ represents the noise vector at $\mathrm{U}_{k}$. The elements of $\boldsymbol{n}_{k}$ are assumed to be i.i.d. $\mathcal{C N}\left(0, \sigma_{n}\right)$.

The transmit vector of $\mathrm{R}$ can be expressed as $\boldsymbol{x}_{R}=$ $\boldsymbol{W}_{h d} \tilde{\boldsymbol{y}}_{R}$ where $\boldsymbol{W}_{h d} \in \mathbb{C}^{M \times M}$ is the beamforming matrix at R.

2) Full-Duplex Relay: In this case, $R$ and the users are all FD. All users have two antennas (one for transmission and one for reception) and $\mathrm{R}$ has $2 M$ antennas ( $M$ for transmission and $M$ for reception). We define $\boldsymbol{G}_{2} \in \mathbb{C}^{M \times 2 K}$ and $\boldsymbol{G}_{3}{ }^{\mathrm{T}} \in \mathbb{C}^{2 K \times M}$ as the channels from the transmission antennas of all users to the reception antenna array of $\mathrm{R}$ and the channel from the transmission antennas of $\mathrm{R}$ to the reception antennas of all users, respectively. $\boldsymbol{G}_{R R}$ $\in \mathbb{C}^{M \times M}$ denotes the EI channel matrix between the transmit and receive arrays of $\mathrm{R}$ with i.i.d $\mathcal{C N}\left(0, \sigma_{r r}^{2}\right)$ elements. $\Psi_{k, k}$ and $\Psi_{k, i}\left(i \in S_{k}, i \neq k\right)$ represent the selfloop interference coefficient at $U_{k}$ and the interuser interference channel coefficient from $U_{i}$ to $U_{k}$, respectively, where $S_{k}=\left\{1,3, \cdots, 2 K^{-1}\right\}$ or $\{2,4$, $\cdots, 2 K$ \} denotes the users on the same side as $\mathrm{U}_{k}$. The elements of $\Psi_{k, k}$ and $\Psi_{k, i}$ can be modeled as i.i.d. $\mathcal{C N}\left(0, \phi_{k, k}\right)$ and $\mathcal{C N}\left(0, \phi_{k, i}\right)$ random variables.

At time instant $t$, all sources transmit their symbols to $\mathrm{R}$ and $\mathrm{R}$ forwards the amplified signal to its destinations. The received signals at the relay and the $k$ th user are given by

$$
\begin{aligned}
\tilde{y}_{R}(t)= & \boldsymbol{y}_{R}(t)+\boldsymbol{r}_{R}(t)=\boldsymbol{G}_{2}\left(\boldsymbol{x}_{s}(t)+\boldsymbol{t}_{s}(t)\right) \\
& +\boldsymbol{G}_{R R}\left(\boldsymbol{x}_{R}(t)+\boldsymbol{t}_{R}(t)\right)+\boldsymbol{n}_{R}(t)+\boldsymbol{r}_{R}(t), \\
\tilde{y}_{k}(t)= & y_{k}(t)+r_{k}(t)=\boldsymbol{g}_{3, k}^{\mathrm{T}}\left[\boldsymbol{x}_{R}(t)+\boldsymbol{t}_{R}(t)\right] \\
& +n_{k}(t)+\sum_{i \in S_{k}} \psi_{k, i} x_{i}(t)+r_{k}(t) .
\end{aligned}
$$

The transmit vector of R at time instant $t$ with power $P_{R}=\mathrm{Tr}$ $\left(\mathrm{E}\left\{\boldsymbol{x}_{R}(t) \boldsymbol{x}_{R}{ }^{\mathrm{H}}(t)\right\}\right)$, can be expressed as $\boldsymbol{x}_{R}(t)=\boldsymbol{W}_{f d} \tilde{\boldsymbol{y}}_{R}(t-d)$, where $\boldsymbol{W}_{f d} \in \mathbb{C}^{M \times M}$ is the beamforming matrix, and $\mathrm{d}$ denotes the processing delay at $\mathrm{R}$.

In this paper, we regard the RLI (Residual Loop Interference) at $\mathrm{R}$ as additional noise ${ }^{[13]}$. As a result, we replace $\boldsymbol{x}_{R}(t)$ in Eq.(3) with a Gaussian noise source $\tilde{\boldsymbol{x}}_{R}(t)$ with the same power limitation to represent the RLI signal. For simplicity, the time labels are omitted in the sequel.

The Rician fading channel matrix above is modeled as $\boldsymbol{G}_{i}=\boldsymbol{H}_{i} \boldsymbol{D}_{i}^{1 / 2}$, where $\boldsymbol{H}_{i} \in \mathbb{C}^{M \times 2 K}(i=1,2$, 3 ) is the channel matrix representing fast fading and $\boldsymbol{D}_{i} \in \mathbb{C}^{2 K \times 2 K}$ is the diagonal matrix representing largescale fading with $\left[\boldsymbol{D}_{i}\right]_{k k}=\beta_{i, k}$. Then, the fast fading matrix can be written as $\boldsymbol{H}_{i}=\overline{\boldsymbol{H}}_{i}\left[\boldsymbol{\Omega}\left(\boldsymbol{\Omega}+\boldsymbol{I}_{2 K}\right)^{-1}\right]^{1 / 2}$ $+\boldsymbol{H}_{i, w}\left[\left(\boldsymbol{\Omega}+\boldsymbol{I}_{2 K}\right)^{-1}\right]^{1 / 2}$, where $\Omega$ is a $2 K \times 2 K$ Rician $K$-factor diagonal matrix with $[\boldsymbol{\Omega}]_{i i}=K_{i}, H_{i, w}$ contains the independent identically distributed (i.i.d.) $\mathcal{C N}(0,1)$ entries. $\overline{\boldsymbol{H}}_{i}$ denotes the deterministic component, and we let $\overline{\boldsymbol{H}}_{i}$ have an arbitrary rank as ${ }^{[14]}\left[\overline{\boldsymbol{H}}_{i}\right]_{m k}=$ $\mathrm{e}^{-\mathrm{j}(m-1)(2 \pi d / \lambda) \sin \left(\theta_{k}\right)}$, where $\theta_{k}$ is the arrival angle of the $k$ th user, $\lambda$ is the wavelength, and $d$ is the antenna spacing. 
For convenience, we will set $d=\lambda / 2$ in this paper.

In this paper, we assume $\mathrm{R}$ has estimates of channels $\boldsymbol{G}_{i}$, but no instantaneous knowledge of $\boldsymbol{G}_{R R}$. For the considered Rician fading channel model, we assume that both the Rician $K$-factor matrix and the deterministic LOS component are perfectly known at R, so we only need to estimate $\boldsymbol{G}_{i, \omega} \triangleq \boldsymbol{H}_{i, \omega} D_{i}^{1 / 2}$. The real channel can be represented as $\boldsymbol{G}_{i}=\hat{\boldsymbol{G}}_{i}+\Delta \boldsymbol{G}_{i}$, where $\hat{\boldsymbol{G}}_{i}$ and $\Delta \boldsymbol{G}_{i}$ denote the available channel estimate and estimation error, respectively. The elements of the $k$ th column of $\Delta \boldsymbol{G}_{i}$ are RVs with zero means and variances $\varepsilon_{i, k}=\xi_{i, k} /\left(K_{k}+1\right)$. Furthermore, $\Delta \boldsymbol{g}_{i, k}$ is independent of $\hat{\boldsymbol{g}}_{i, k}$ if $\hat{\boldsymbol{g}}_{i, k}$ is the MMSE estimation of $\boldsymbol{g}_{i, k}$. In this paper, we consider $\Delta \boldsymbol{g}_{i, k}$ and $\hat{\boldsymbol{g}}_{i, k}$ to be independent.

\section{Relay transceiver design and asymptotic analysis}

In this section, we propose a ZFR/ZFT beamforming design. The asymptotic end-to-end SINR of the proposed transceiver scheme for multipair two-way HDR and FDR system are analyzed.

Lemma 1 By the law of large numbers, when $M$ is large enough, the inner product of any two columns in the estimate channel matrix $\hat{G}_{i}$ can be found as ${ }^{[14]}$

$$
\begin{aligned}
& \frac{\hat{g}_{i, n}^{\mathrm{H}} \hat{g}_{i, j}}{M} \stackrel{\text { a.s. }}{\longrightarrow} \begin{cases}\frac{\beta_{i, n} K_{n}}{K_{n}+1}+\frac{\beta_{i, n}-\xi_{i, n}}{K_{n}+1}=\eta_{i, n}, & j=n \\
0, & j \neq n\end{cases} \\
& \frac{\hat{G}_{i}^{\mathrm{H}} \hat{G}_{i}}{M} \stackrel{\text { a.s. }}{\longrightarrow} \operatorname{diag}\left\{\eta_{i, 1}, \eta_{i, 2}, \cdots, \eta_{i, 2 K}\right\}=\boldsymbol{Q}_{i} .
\end{aligned}
$$

1) Half-Duplex Relay: The ZFR/ZFT beamforming matrix is

$$
\boldsymbol{W}_{h d}=a_{z f} \hat{\boldsymbol{G}}_{1}^{*}\left(\hat{\boldsymbol{G}}_{1}^{\mathrm{T}} \hat{\boldsymbol{G}}_{1}^{*}\right)^{-1} \boldsymbol{P}\left(\hat{\boldsymbol{G}}_{1}^{\mathrm{T}} \hat{\boldsymbol{G}}_{1}\right)^{-1} \hat{\boldsymbol{G}}_{1}^{\mathrm{T}},
$$

where $a_{z f}$ is the amplification factor, $\boldsymbol{P}=\operatorname{diag}\left\{\boldsymbol{P}_{1}\right.$, $\left.\boldsymbol{P}_{2}, \cdots, \boldsymbol{P}_{K}\right\}$ and $\boldsymbol{P}_{l}=[01 ; 10] ; l=1, \cdots, K$.

Lemma 2 As $M$ approaches infinity, $a_{z f}$ that satisfies the transmit power constraint of $\mathrm{R}$ can be expressed as

$$
\sqrt{\frac{a_{z j} \stackrel{a . s .}{\longrightarrow}}{M \sum_{i=1}^{2 K}\left(1+v_{i}\right) P_{s, j} \eta_{1, i^{\prime}}^{-1}+\left[\sum_{j=1}^{2 K}\left(1+v_{j}\right) P_{s, j} \varepsilon_{1, j}+\sigma+P_{R}^{r}\right] \sum_{i=1}^{2 K} \eta_{1, i}^{-1} \eta_{1, i^{\prime}}^{-1}}},
$$

where $P_{R}^{r}=\mu_{R}\left(\sum_{i=1}^{2 K}\left(1+v_{i}\right) P_{s, i} \beta_{1, i}+\sigma\right),\left(i ; i^{\prime}\right)$ is a pair of user. See the proof in Appendix A).

Substituting $\boldsymbol{g}_{1, k}=\hat{\boldsymbol{g}}_{1, k}+\Delta \boldsymbol{g}_{1, k}, \boldsymbol{G}_{1}=\hat{\boldsymbol{G}}_{1}+\Delta \boldsymbol{G}_{1}$ and Eq.(6) into Eq.(2), the received signal at the $k$ th user is

$$
\begin{aligned}
& \tilde{y}_{k}=a_{z f} x_{k^{\prime}}+y^{\prime 1}+y^{n}+y^{r}+y^{\prime 2}+n_{k}+r_{k}, \\
& y^{t 1}=a_{z f} \mathbf{1}_{k^{\prime}} \boldsymbol{t}_{s}+a_{z f} \mathbf{1}_{k^{\prime}}\left(\hat{\boldsymbol{G}}_{1}^{\mathrm{H}} \hat{\boldsymbol{G}}_{1}\right)^{-1} \hat{\boldsymbol{G}}_{1}^{\mathrm{H}} \Delta \boldsymbol{G}_{1}\left(\boldsymbol{x}_{s}+\boldsymbol{t}_{s}\right) \\
& +a_{z f} \Delta \boldsymbol{g}_{1, k}^{\mathrm{T}} \hat{\boldsymbol{G}}_{1}^{*}\left(\hat{\boldsymbol{G}}_{1}^{\mathrm{T}} \hat{\boldsymbol{G}}_{1}^{*}\right)^{-1} \boldsymbol{P}\left(\boldsymbol{x}_{s}+\boldsymbol{t}_{s}\right) \\
& +a_{z f} \Delta \boldsymbol{g}_{1, k}^{\mathrm{T}} \hat{\boldsymbol{G}}_{1}^{*}\left(\hat{\boldsymbol{G}}_{1}^{\mathrm{T}} \hat{\boldsymbol{G}}_{1}^{*}\right)^{-1} \boldsymbol{P}\left(\hat{\boldsymbol{G}}_{1}^{\mathrm{H}} \hat{\boldsymbol{G}}_{1}\right)^{-1} \hat{\boldsymbol{G}}_{1}^{\mathrm{H}} \Delta \boldsymbol{G}_{1}\left(\boldsymbol{x}_{s}+\boldsymbol{t}_{s}\right), \\
& y^{n}=a_{z f} \mathbf{1}_{k^{\prime}}\left(\hat{\boldsymbol{G}}_{1}^{\mathrm{H}} \hat{\boldsymbol{G}}_{1}\right)^{-1} \hat{\boldsymbol{G}}_{1}^{\mathrm{H}} \boldsymbol{n}_{R} \\
& +a_{z f} \Delta \boldsymbol{g}_{1, k}^{\mathrm{T}} \hat{\boldsymbol{G}}_{1}^{*}\left(\hat{\boldsymbol{G}}_{1}^{\mathrm{T}} \hat{\boldsymbol{G}}_{1}^{*}\right)^{-1} \boldsymbol{P}\left(\hat{\boldsymbol{G}}_{1}^{\mathrm{H}} \hat{\boldsymbol{G}}_{1}\right)^{-1} \hat{\boldsymbol{G}}_{1}^{\mathrm{H}} \boldsymbol{n}_{R}, \\
& y^{r}=a_{z f} \mathbf{1}_{k^{\prime}}\left(\hat{\boldsymbol{G}}_{1}^{\mathrm{H}} \hat{\boldsymbol{G}}_{1}\right)^{-1} \hat{\boldsymbol{G}}_{1}^{\mathrm{H}} \boldsymbol{r}_{R} \\
& +a_{z f} \Delta \boldsymbol{g}_{1, k}^{\mathrm{T}} \hat{\boldsymbol{G}}_{1}^{*}\left(\hat{\boldsymbol{G}}_{1}^{\mathrm{T}} \hat{\boldsymbol{G}}_{1}^{*}\right)^{-1} \boldsymbol{P}\left(\hat{\boldsymbol{G}}_{1}^{\mathrm{H}} \hat{\boldsymbol{G}}_{1}\right)^{-1} \hat{\boldsymbol{G}}_{1}^{\mathrm{H}} \boldsymbol{r}_{R}, \\
& y^{\prime 2}=\left(\hat{\boldsymbol{g}}_{1, k}^{\mathrm{T}}+\Delta \boldsymbol{g}_{1, k}^{\mathrm{T}}\right) \boldsymbol{t}_{R}
\end{aligned}
$$

where $\mathbf{1}_{k^{\prime}}$ represents a $1 \times 2 K$ vector, in which the $k^{\prime}$ th entry is 1 and the others are all 0 .

Theorem 1 Using a ZFR/ZFT beamforming matrix with imperfect CSI, the end-to-end SINR of HDR system at the $k$ th user can be expressed asymptotically (in $M$ ) as

$$
\gamma_{k}=\frac{P_{s, k^{\prime}} a_{z f}^{2}}{\mathrm{E}\left[\left|y^{t 1}\right|^{2}+\left|y^{n}\right|^{2}+\left|y^{r}\right|^{2}+\left|y^{\prime 2}\right|^{2}+\sigma_{n}+r_{k} r_{k}^{\mathrm{H}}\right]},
$$

where the power for the terms in the denominator can be expressed asymptotically (in $M$ ) as

$\mathrm{E}\left[\left|y^{t 1}\right|^{2}\right]=a_{z f}^{2}\left[v_{k^{\prime}} P_{s, k^{\prime}}+\frac{\eta_{1, k^{\prime}}^{-1}}{M} \sum_{i=1}^{2 K}\left(1+v_{i}\right) P_{s, i} \varepsilon_{1, i}+\frac{\varepsilon_{1, k}}{M}\right.$

$\left.\cdot \sum_{i=1}^{2 K}\left(1+v_{i}\right) P_{s, i} \eta_{1, i^{\prime}}^{-1}+\frac{\varepsilon_{1, k}}{M^{2}} \sum_{i=1}^{2 K}\left(1+v_{i}\right) P_{s, i} \varepsilon_{1, i} \sum_{i=1}^{2 K} \eta_{1, i}^{-1} \eta_{1, i^{\prime}}^{-1}\right]$,

$\mathrm{E}\left[\left|y^{n}\right|^{2}\right]=a_{z f}^{2} \sigma\left[\frac{\eta_{1, k^{\prime}}^{-1}}{M}+\frac{\varepsilon_{1, k}}{M^{2}} \sum_{i=1}^{2 K} \eta_{1, i}^{-1} \eta_{1, i^{\prime}}^{-1}\right]$, 


$$
\begin{aligned}
& \mathrm{E}\left[\left|y^{r}\right|^{2}\right]=a_{z f}^{2} P_{R}^{r}\left[\frac{\eta_{1, k^{\prime}}^{-1}}{M}+\frac{\varepsilon_{1, k}}{M^{2}} \sum_{i=1}^{2 K} \eta_{1, i}^{-1} \eta_{1, i^{\prime}}^{-1}\right], \\
& \mathrm{E}\left[\left|y^{\prime 2}\right|^{2}\right]=v_{R} P_{R} \beta_{1, k}, \\
& \mathrm{E}\left[\left|r_{k} r_{k}^{\mathrm{H}}\right|\right]=\mu_{k}\left\{a _ { z f } ^ { 2 } \left[\left(1+v_{k^{\prime}}\right) P_{s, k^{\prime}}+\frac{\eta_{1, k^{\prime}}^{-1}}{M} \sum_{i=1}^{2 K}\left(1+v_{i}\right) P_{s, i} \varepsilon_{1, i}\right.\right. \\
& \left.+\frac{\varepsilon_{1, k}}{M} \sum_{i=1}^{2 K}\left(1+v_{i}\right) P_{s, i} \eta_{1, i^{\prime}}^{-1}+\frac{\varepsilon_{1, k}}{M^{2}} \sum_{i=1}^{2 K}\left(1+v_{i}\right) P_{s, i^{\prime}} \varepsilon_{1, i} \sum_{i=1}^{2 K} \eta_{1, i}^{-1} \eta_{1, i^{\prime}}^{-1}\right] \\
& \left.+a_{z f}^{2}\left[\frac{\eta_{1, k^{\prime}}^{-1}}{M}+\frac{\varepsilon_{1, k}}{M^{2}} \sum_{i=1}^{2 K} \eta_{1, i}^{-1} \eta_{1, i^{\prime}}^{-1}\right]\left[\sigma+P_{R}^{r}\right]+v_{R} P_{R} \beta_{1, k}+\sigma_{n}\right\} .
\end{aligned}
$$

See the proof in Appendix B).

Theorem 1 reveals that, when using very large antenna arrays, the SINR of a HDR system is limited by the effective distortion noise caused by the imperfect transmit and receive RF chains of the users. More precisely, we can see that even very small imperfections in the transmit and receive RF chains of the users can degrade the SINR significantly. This is because the value of the denominator in Eq.(9) mainly depends on $a_{z f}^{2} v_{k^{\prime}} P_{s, k^{\prime}}$ and $\mu_{k} a_{z f}^{2}\left(1+v_{k^{\prime}}\right) P_{s, k^{\prime}}$, which scale as $\mathcal{O}(M)$ when $M$ approaches to infinity, while other terms of the denominator scale as $\mathcal{O}(1)$. Thus, the imperfection of the users is $M$ times more influential than the hardware impairments of $\mathrm{R}$.

2) Full-Duplex Relay: The ZFR/ZFT beamforming matrix is

$$
\boldsymbol{W}_{f d}=a_{z f^{\prime}} \hat{\boldsymbol{G}}_{3}^{*}\left(\hat{\boldsymbol{G}}_{3}^{\mathrm{T}} \hat{\boldsymbol{G}}_{3}^{*}\right)^{-1} \boldsymbol{P}\left(\hat{\boldsymbol{G}}_{2}^{\mathrm{H}} \hat{\boldsymbol{G}}_{2}\right)^{-1} \hat{\boldsymbol{G}}_{2}^{\mathrm{H}},
$$

where $a_{z f}$ is the amplification factor.

Lemma 3 As $M$ approaches infinity, $a_{z f}$, that satisfies the transmit power constraint of $\mathrm{R}$ can be expressed as

$$
\begin{aligned}
a_{z f^{\prime}} \stackrel{a . s .}{\longrightarrow} & \left(P_{R} M^{2} /\left\{M \sum_{i=1}^{2 K}\left(1+v_{i}\right) P_{s, i} \eta_{3, i^{\prime}}^{-1}\right.\right. \\
& +\left[\sum_{j=1}^{2 K}\left(1+v_{j}\right) P_{s, j} \varepsilon_{2, j}+\sigma_{r r}^{2}\left(1+v_{R}\right) P_{R}\right. \\
& \left.\left.\left.+\sigma+P_{R}^{r 1}\right] \sum_{i=1}^{2 K} \eta_{2, i}^{-1} \eta_{3, i^{\prime}}^{-1}\right\}\right)^{1 / 2}
\end{aligned}
$$

where $P_{R}^{r}=\mu_{R}\left(\sum_{i=1}^{2 K}\left(1+v_{i}\right) P_{s, i} \beta_{2, i}+\sigma_{r r}^{2}\left(1+v_{R}\right) P_{R}+\sigma\right)$.

See the proof in Appendix C).

Substituting $\boldsymbol{g}_{3, k}=\hat{\boldsymbol{g}}_{3, k}+\Delta \boldsymbol{g}_{3, k}, \boldsymbol{G}_{2}=\hat{\boldsymbol{G}}_{2}+\Delta \boldsymbol{G}_{2}$ and Eq.(11) into Eq.(4), the received signal at the $k$ th user is

$$
\begin{aligned}
& \tilde{y}_{k}=a_{z f^{\prime}} x_{k^{\prime}}+y^{t 1}+y^{L 1}+y^{n}+y^{r} \\
& +y^{t 2}+\sum_{i \in S_{k}} \psi_{k, i} x_{i}+n_{k}+r_{k}, \\
& y^{\prime 1}=a_{z f^{\prime}} \mathbf{1}_{k^{\prime}} \boldsymbol{t}_{s}+a_{z f^{\prime}} \mathbf{1}_{k^{\prime}}\left(\hat{\boldsymbol{G}}_{2}^{\mathrm{H}} \hat{\boldsymbol{G}}_{2}\right)^{-1} \hat{\boldsymbol{G}}_{2}^{\mathrm{H}} \Delta \boldsymbol{G}_{2}\left(\boldsymbol{x}_{s}+\boldsymbol{t}_{s}\right) \\
& +\Delta \boldsymbol{g}_{3, k}^{\mathrm{T}} a_{z f^{\prime}} \hat{\boldsymbol{G}}_{3}^{*}\left(\hat{\boldsymbol{G}}_{3}^{\mathrm{T}} \hat{\boldsymbol{G}}_{3}^{*}\right)^{-1} \boldsymbol{P}\left(\boldsymbol{x}_{s}+\boldsymbol{t}_{s}\right) \\
& +\Delta \boldsymbol{g}_{3, k}^{\mathrm{T}} a_{z f^{\prime}} \hat{\boldsymbol{G}}_{3}^{*}\left(\hat{\boldsymbol{G}}_{3}^{\mathrm{T}} \hat{\boldsymbol{G}}_{3}^{*}\right)^{-1} \boldsymbol{P}\left(\hat{\boldsymbol{G}}_{2}^{\mathrm{H}} \hat{\boldsymbol{G}}_{2}\right)^{-1} \hat{\boldsymbol{G}}_{2}^{\mathrm{H}} \Delta \boldsymbol{G}_{2}\left(\boldsymbol{x}_{s}+\boldsymbol{t}_{s}\right), \\
& g^{L I}=a_{z f^{\prime}} \mathbf{1}_{k^{\prime}}\left(\hat{\boldsymbol{G}}_{2}^{\mathrm{H}} \hat{\boldsymbol{G}}_{2}\right)^{-1} \hat{\boldsymbol{G}}_{2}^{\mathrm{H}} \boldsymbol{G}_{R R}\left(\tilde{\boldsymbol{x}}_{R}+\boldsymbol{t}_{R}\right) \\
& +a_{z f^{\prime}}, \Delta \boldsymbol{g}_{3, k}^{\mathrm{T}} \hat{\boldsymbol{G}}_{3}^{*}\left(\hat{\boldsymbol{G}}_{3}^{\mathrm{T}} \hat{\boldsymbol{G}}_{3}^{*}\right)^{-1} \boldsymbol{P}\left(\hat{\boldsymbol{G}}_{2}^{\mathrm{H}} \hat{\boldsymbol{G}}_{2}\right)^{-1} \hat{\boldsymbol{G}}_{2}^{\mathrm{H}} \boldsymbol{G}_{R R}\left(\tilde{\boldsymbol{x}}_{R}+\boldsymbol{t}_{R}\right), \\
& y^{n}=a_{z f^{\prime}} \mathbf{1}_{k^{\prime}}\left(\hat{\boldsymbol{G}}_{2}^{\mathrm{H}} \hat{\boldsymbol{G}}_{2}\right)^{-1} \hat{\boldsymbol{G}}_{2}^{\mathrm{H}} \boldsymbol{n}_{R} \\
& +a_{z f^{\prime}} \Delta \boldsymbol{g}_{3, k}^{\mathrm{T}} \hat{\boldsymbol{G}}_{3}^{*}\left(\hat{\boldsymbol{G}}_{3}^{\mathrm{T}} \hat{\boldsymbol{G}}_{3}^{*}\right)^{-1} \boldsymbol{P}\left(\hat{\boldsymbol{G}}_{2}^{\mathrm{H}} \hat{\boldsymbol{G}}_{2}\right)^{-1} \hat{\boldsymbol{G}}_{2}^{\mathrm{H}} \boldsymbol{n}_{R}, \\
& y^{r}=a_{z f^{\prime}} \mathbf{1}_{k^{\prime}}\left(\hat{\boldsymbol{G}}_{2}^{\mathrm{H}} \hat{\boldsymbol{G}}_{2}\right)^{-1} \boldsymbol{G}_{2}^{\mathrm{H}} \boldsymbol{r}_{R} \\
& +a_{z f^{\prime}} \Delta \boldsymbol{g}_{3, k}^{\mathrm{T}} \hat{\boldsymbol{G}}_{3}^{*}\left(\hat{\boldsymbol{G}}_{3}^{\mathrm{T}} \hat{\boldsymbol{G}}_{3}^{*}\right)^{-1} \boldsymbol{P}\left(\hat{\boldsymbol{G}}_{2}^{\mathrm{H}} \hat{\boldsymbol{G}}_{2}\right)^{-1} \hat{\boldsymbol{G}}_{2}^{\mathrm{H}} \boldsymbol{r}_{R}, \\
& y^{\prime 2}=\left(\hat{\boldsymbol{g}}_{3, k}^{\mathrm{T}}+\Delta \boldsymbol{g}_{3, k}^{\mathrm{T}}\right) \boldsymbol{t}_{R} .
\end{aligned}
$$

Theorem 2 Using a ZFR/ZFT beam forming matrix with imperfect CSI, the end-to-end SINR of a FDR system at the $k$ th user can be expressed asymptotically (in $M$ ) as

$$
\begin{aligned}
\gamma_{k}= & P_{s, k^{\prime}} a_{z f^{\prime}}^{2} / \mathrm{E}\left(\left|y^{t 1}\right|^{2}+\left|y^{L I}\right|^{2}+\left|y^{n}\right|^{2}\right. \\
& \left.+\left|y^{r}\right|^{2}+\left|y^{t 2}\right|^{2}+\sum_{i \in S_{k}} \phi_{k, i} P_{s, i}+\sigma_{n}+r_{k} r_{k}^{\mathrm{H}}\right),
\end{aligned}
$$

where the power for the terms in the denominator can be expressed asymptotically (in $M$ ) as

$$
\begin{aligned}
& \mathrm{E}\left[\left|y^{t 1}\right|^{2}\right]=a_{z f^{\prime}}^{2}\left[v_{k} P_{s, k^{\prime}}+\frac{\eta_{2, k^{\prime}}^{-1}}{M} \sum_{i=1}^{2 K}\left(1+v_{i}\right) P_{s, i} \varepsilon_{2, i}\right. \\
& \left.+\frac{\varepsilon_{3, k}}{M} \sum_{i=1}^{2 K}\left(1+v_{i}\right) P_{s, i} \eta_{3, i^{\prime}}^{-1}+\frac{\varepsilon_{3, k}}{M^{2}} \sum_{j=1}^{2 K}\left(1+v_{j}\right) P_{s, j} \varepsilon_{2, j} \sum_{i=1}^{2 K} \eta_{2, i}^{-1} \eta_{3, i^{\prime}}^{-1}\right], \\
& \mathrm{E}\left[\left|y^{L I}\right|^{2}\right]=a_{z f^{\prime}}^{2} \sigma_{r r}^{2}\left(1+v_{R}\right) P_{R}\left[\frac{\eta_{2, k^{\prime}}^{-1}}{M}+\frac{\varepsilon_{3, k}}{M^{2}} \sum_{i=1}^{2 K} \eta_{2, i}^{-1} \eta_{3, i^{\prime}}^{-1}\right],
\end{aligned}
$$




$$
\begin{aligned}
& \mathrm{E}\left[\left|y^{n}\right|^{2}\right]=a_{z f^{\prime}}^{2} \sigma\left[\frac{\eta_{2, k^{\prime}}^{-1}}{M}+\frac{\varepsilon_{3, k}}{M^{2}} \sum_{i=1}^{2 K} \eta_{2, i}^{-1} \eta_{3, i^{\prime}}^{-1}\right], \\
& \mathrm{E}\left[\left|y^{r}\right|^{2}\right]=a_{z f^{\prime}}^{2} P_{R}^{r 1}\left[\frac{\eta_{2, k^{\prime}}^{-1}}{M}+\frac{\varepsilon_{3, k}}{M^{2}} \sum_{i=1}^{2 K} \eta_{2, i}^{-1} \eta_{3, i^{\prime}}^{-1}\right], \\
& \mathrm{E}\left[\left|y^{\prime 2}\right|^{2}\right]=v_{R} P_{R} \beta_{3, k}, \\
& \mathrm{E}\left[\left|r_{k} r_{k}^{\mathrm{H}}\right|\right]=\mu_{k}\left\{a _ { z f ^ { \prime } } ^ { 2 } \left[\left(1+v_{k^{\prime}}\right) P_{s, k^{\prime}}+\frac{\eta_{2, k^{\prime}}^{-1}}{M} \sum_{i=1}^{2 K}\left(1+v_{i}\right) P_{s, i} \varepsilon_{2, i}\right.\right. \\
& \left.+\frac{\varepsilon_{3, k}}{M} \sum_{i=1}^{2 K}\left(1+v_{i}\right) P_{s, i} \eta_{3, i^{\prime}}^{-1}+\frac{\varepsilon_{3, k}}{M^{2}} \sum_{j=1}^{2 K}\left(1+v_{j}\right) P_{s, j} \varepsilon_{2, j} \sum_{i=1}^{2 K} \eta_{2, i^{-1}} \eta_{3, i^{\prime}}^{-1}\right] \\
& +a_{z f^{\prime}}^{2}\left[\frac{\eta_{2, k^{\prime}}^{-1}}{M}+\frac{\varepsilon_{3, k}}{M^{2}} \sum_{i=1}^{2 K} \eta_{2, i^{\prime}}^{-1} \eta_{3, i^{\prime}}^{-1}\right]\left[\sigma_{r r}^{2}\left(1+v_{R}\right) P_{R}+\sigma+P_{R}^{r 1}\right] \\
& \left.+v_{R} P_{R} \beta_{3, k}+\sum_{i \in S_{k}} \phi_{k, i} P_{s, i}+\sigma_{n}\right\} .
\end{aligned}
$$

See the proof in Appendix D).

A key result from Theorem 2 is that the limiting factor for the SINR of a FDR system in a large M regime is not the hardware impairment of $\mathrm{R}$ or other interferences, but the effective distortion noise caused by the imperfect transmit and receive RF chains of the users. In fact, even very small imperfections in the transmit and receive RF chains of the users can significantly degrade the SINR. This is because the value of the denominator in Eq.(14) mainly depends on $a_{z f^{\prime}}^{2} v_{k^{\prime}} P_{s, k^{\prime}}$ and $\mu_{k} a_{z f^{\prime}}^{2}\left(1+v_{k^{\prime}}\right) P_{s, k^{\prime}}$, which scale as $\mathcal{O}(M)$ when $\mathrm{M}$ approaches infinity, while other terms of the denominator scale as $\mathcal{O}(1)$. Thus, the hardware impairments of the users become dominant as $M$ approaches infinity.

\section{Simulation results}

In this section, we examine the SE of the multipair two-way HDR and FDR system which are defined as $S E_{h d}=\mathrm{E}\left[\sum_{i=1}^{2 K} \mathrm{lb}\left(1+\gamma_{i}\right)\right] / 2$ and $S E_{f d}=\mathrm{E}\left[\sum_{i=1}^{2 K} \mathrm{lb}\left(1+\gamma_{i}\right)\right]$, respectively. Without a loss of generality, we assume that $\sigma=\sigma_{n}=\phi_{k, i}=1, \boldsymbol{D}_{i}=\boldsymbol{I}_{2 K}, \xi_{i, n}=\xi, P_{s, k}=P_{s}, v_{k}=$ $v_{s}, \mu_{k}=\mu_{s}(k=1, \cdots, 2 K)$, and all users have the same Rician K-factor $K_{i}$. In order to make the comparison more fair, the transmit powers in HDR are set as $2 P_{s}$ and $2 P_{R}$ (two times of FDR) for all nodes transmit only half of the time in HDR.

Fig. 1 shows the SE of a multipair HDR and FDR v.s. $M$ for different levels of hardware impairment. We can see that the asymptotic expression derived in Theorems 1 and 2 can predict the performance of the multipair HDR and FDR precisely as $M$ increases. Moreover, the SE can be greatly improved as high-quality hardware is used at the users $\left(v_{s}\right.$ and $\mu_{s}$ decrease from 0.05 to 0.001 ), especially in a large $M$ scenario. However, the effect of hardware impairments at $\mathrm{R}\left(v_{R}\right.$ and $\mu_{R}$ increase from 0.001 to $0.05)$ is insignificant. This matches well with the conclusion drawn in section III. In addition, it is seen that the FDR outperforming HDR in the large $M$ scenario.

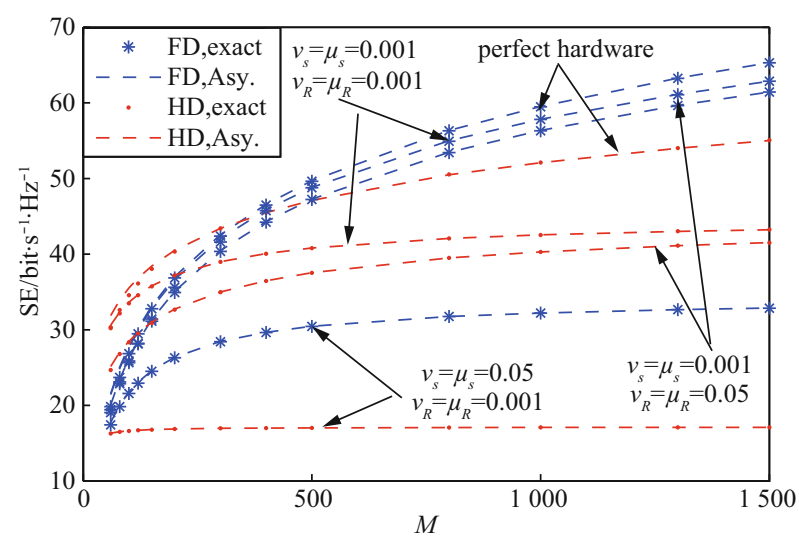

Figure $1 \mathrm{SE}$ of multipair FDR and HDR vs. $M$, where $K=$ $5, K_{i}=4, P_{s}=5 \mathrm{~dB}, P_{R}=2 K P_{s}, \xi=0.1$, and $\sigma_{r r}^{2}=1$

From Fig.2, we can see that there exists a switching point between HDR and FDR as $\sigma_{r r}^{2}$ increases. By increasing $M$, the constraint on $\sigma_{r r}^{2}$ for FDR outperforming HDR relaxes, which implies that FDR becomes attractive when $M$ is large. This is because the effect of EI in FDR becomes smaller in this case. 


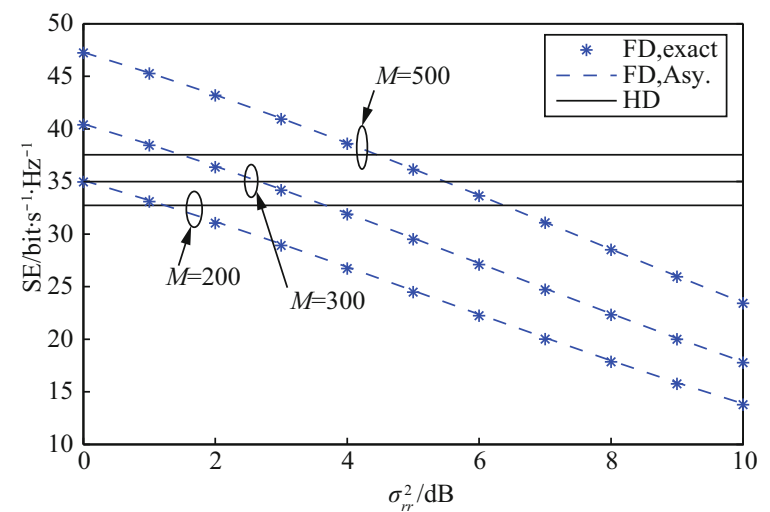

Figure $2 \mathrm{SE}$ of multipair FDR and HDR v.s. $\sigma_{r r}^{2}$, where $K$ $=5, K_{i}=4, P_{s}=5 \mathrm{~dB}, P_{R}=2 K P_{s}, \xi=0.1, v_{s}=\mu_{s}=0.001$, and $v_{R}=\mu_{R}=0.05$

Fig. 3 depicts the SE loss of multipair FDR and HDR with perfect and imperfect hardware under different Rician $K$-factors. We denote the SE loss between perfect hardware (ph) and imperfect hardware(iph) as $S E_{\text {loss }}=\left(S E_{\mathrm{ph}}-S E_{\mathrm{iph}}\right) / S E_{\mathrm{ph}}$. Clearly, the SE loss increases as $K_{i}$ increases, which reveals that the favorable propagation in multipair FDR and HDR systems can mitigate the influence of hardware impairments. In addition, the SE loss in multi-pair HDR is larger than FDR.
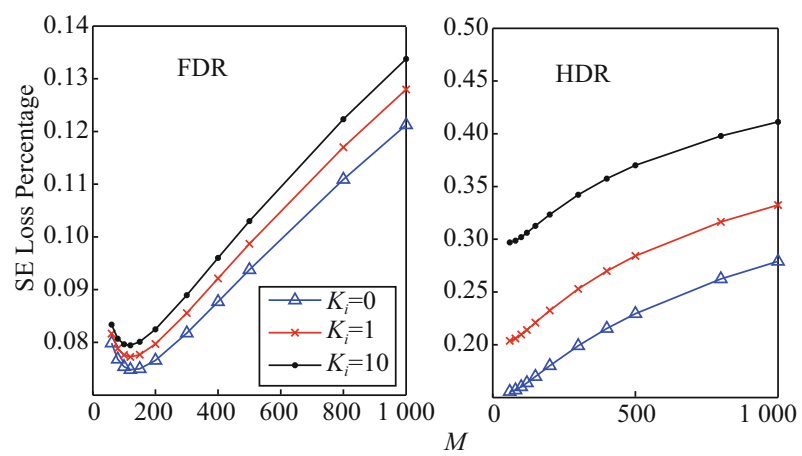

Figure 3 SE loss of multipair FDR and HDR against $M$ and $K_{i}$, where $K=5, P_{s}=5 \mathrm{~dB}, P_{R}=2 K P_{s}, \xi=0.1, v_{s}=\mu_{s}$ $=0.005, v_{R}=\mu_{R}=0.05$, and $\sigma_{r r}^{2}=1$

\section{Conclusion}

In this paper, we investigated a multipair two-way massive MIMO AF relay system over HAPs with hardware impairments in the case of imperfect CSI. The ZFR/ZFT processing matrices of HDR and FDR are presented and the asymptotic expressions in $\mathrm{M}$ of the end-to-end SINR are derived analytically. Theoretical analyses and simulation results show that, when $M$ is large enough, the SE of a massive MIMO AF relay system is not sensitive to the hardware impairments at $\mathrm{R}$. This implies that the quality of hardware at $\mathrm{R}$ can be decreased without significantly degrading the performance in a highaltitude platform. Moreover, it is shown that the multipair FDR outperforms the multipair HDR in a large $M$ scenario. Finally, we conclude that the SE loss resulting from hardware impairments increases as the Rician K-factor increases. Therefore, it is more important to utilize better-quality hardware when operating in a strong LoS environment.

\section{Appendix}

A) Proof of Eq.(7) in Lemma 2

We denote $\mathrm{E}\left\{t_{s} t_{s}^{\mathrm{H}}\right\}=\operatorname{diag}\left(v_{1} P_{s, 1}, \cdots, v_{2 K} P_{s, 2 K}\right)=$ $\Lambda_{1}, \mathrm{E}\left\{\left(\boldsymbol{x}_{s}+t_{s}\right)\left(\boldsymbol{x}_{s}+t_{s}\right)^{\mathrm{H}}\right\}=\operatorname{diag}\left(\left(1+v_{1}\right) P_{s, 1}, \cdots,(1+\right.$ $\left.v_{2 K}\right) P_{s, 2 K}=\boldsymbol{A}$, then

$$
\begin{aligned}
\mathbb{E}\left\{r_{R} r_{R}^{\mathrm{H}}\right\} & =\mu_{R} \operatorname{diag}\left(\mathrm{E}\left[y_{R} y_{R}^{\mathrm{H}}\right]\right) \\
& =\mu_{R} \operatorname{diag}\left(\mathrm{E}\left[\boldsymbol{G}_{1} \boldsymbol{A} \boldsymbol{G}_{1}^{\mathrm{H}}+\sigma \boldsymbol{I}_{M}\right]\right) \\
& =\mu_{R}\left(\sum_{i=1}^{2 K}\left(1+v_{i}\right) P_{s, i} \beta_{1, i}+\sigma\right) \boldsymbol{I}_{M}=P_{R}^{r} \boldsymbol{I}_{M} .
\end{aligned}
$$

Using the property $\operatorname{Tr}(\boldsymbol{A B})=\operatorname{Tr}(\boldsymbol{B} \boldsymbol{A})$ and $P_{R}=\operatorname{Tr}(\mathrm{E}$ $\left.\left\{x_{R} x_{R}{ }^{\mathrm{H}}\right\}\right)$, we have

$$
\begin{aligned}
a_{z f}^{2}= & P_{R} /\left(\operatorname { T r E } \left(\left\{\left(\hat{\boldsymbol{G}}_{1}^{\mathrm{T}} \hat{\boldsymbol{G}}_{1}^{*}\right)^{-1} \boldsymbol{P} \boldsymbol{A} \boldsymbol{P}+\left(\sigma+P_{R}^{r}\right)\right.\right.\right. \\
& \left.\left.\left.\left(\hat{\boldsymbol{G}}_{1}^{\mathrm{T}} \hat{\boldsymbol{G}}_{1}^{*}\right)^{-1} \boldsymbol{P}\left(\hat{\boldsymbol{G}}_{1}^{\mathrm{H}} \hat{\boldsymbol{G}}_{1}\right)^{-1} \boldsymbol{P}+\Delta \boldsymbol{I}\right\}\right)\right),
\end{aligned}
$$
where $\Delta \boldsymbol{I}=\left(\hat{\boldsymbol{G}}_{1}^{\mathrm{T}} \hat{\boldsymbol{G}}_{1}\right)^{-1} \boldsymbol{P}\left(\hat{\boldsymbol{G}}_{1}^{\mathrm{H}} \hat{\boldsymbol{G}}_{1}\right)^{-1} \hat{\boldsymbol{G}}_{1}^{\mathrm{H}} \Delta \boldsymbol{G}_{1} \boldsymbol{A} \Delta \boldsymbol{G}_{1}^{\mathrm{H}} \hat{\boldsymbol{G}}_{1} \cdot\left(\hat{\boldsymbol{G}}_{1}^{\mathrm{H}}\right.$ $\left.\hat{\boldsymbol{G}}_{1}\right)^{-1} \boldsymbol{P}$. Substituting Eq.(5) into Eq.(17), we have

$$
\begin{aligned}
a_{z f}^{2} \stackrel{\text { a.s. }}{\longrightarrow} & P_{R} / \operatorname{Tr}\left\{\frac{\left(Q_{1}\right)^{-1} \boldsymbol{P} \boldsymbol{A P}}{M}\right. \\
& \left.+\left(\sigma+P_{R}^{r}+\sum_{j=1}^{2 K}\left(1+v_{j}\right) P_{s, j} \varepsilon_{1, j}\right) \frac{\left[\left(Q_{1}\right)^{-1} \boldsymbol{P}\right]^{2}}{M^{2}}\right\} .
\end{aligned}
$$




$$
\begin{aligned}
& \operatorname{Tr}\left\{\left(\boldsymbol{Q}_{1}\right)^{-1} \boldsymbol{P} \boldsymbol{A}\right\}=\sum_{i=1}^{2 K}\left(1+v_{i}\right) P_{s, i} \eta_{1, i^{\prime}}^{-1} \\
& \operatorname{Tr}\left\{\left(\boldsymbol{Q}_{1}\right)^{-1} \boldsymbol{P}\right\}^{2}=\sum_{i=1}^{2 K} \eta_{1, i}^{-1} \eta_{1, i^{\prime}}^{-1},
\end{aligned}
$$

then Eq.(7) can be easily obtained.

B) Proof of Theorem 1

$\mathrm{E}\left[\left|1_{k^{\prime}} t_{s}\right|^{2}\right]=\mathrm{E}\left[1_{k^{\prime}} \Lambda_{1} 1_{k^{\prime}}{ }^{\mathrm{T}}\right]=v_{k^{\prime}} P_{s, k^{\prime}}$

Compute E[ $\left[\left.y^{t 1}\right|^{2}\right]$ : According to Eqs.(5), (19), and with property $\hat{\boldsymbol{G}}_{1}$ and $\Delta \boldsymbol{G}_{1}$ are independent, we have

$\mathrm{E}\left[\left|y^{\prime l}\right|^{2}\right]=a_{z f}^{2} \mathrm{E}\left[\mathbf{1}_{k^{\prime}}\left(\hat{\boldsymbol{G}}_{1}^{\mathrm{H}} \hat{\boldsymbol{G}}_{1}\right)^{-1} \hat{\boldsymbol{G}}_{1}^{\mathrm{H}} \Delta \boldsymbol{G}_{1} \boldsymbol{A} \Delta \boldsymbol{G}_{1}^{\mathrm{H}} \hat{\boldsymbol{G}}_{1}\right.$

$\cdot\left(\hat{\boldsymbol{G}}_{1}^{\mathrm{H}} \hat{\boldsymbol{G}}_{1}\right)^{-1} \mathbf{1}_{k^{\prime}}^{\mathrm{T}}+\Delta \boldsymbol{g}_{1, k}^{\mathrm{T}} \hat{\boldsymbol{G}}_{1}^{*}\left(\hat{\boldsymbol{G}}_{1}^{\mathrm{T}} \hat{\boldsymbol{G}}_{1}^{*}\right)^{-1} \boldsymbol{P} \boldsymbol{A} \boldsymbol{P}\left(\hat{\boldsymbol{G}}_{1}^{\mathrm{T}} \hat{\boldsymbol{G}}_{1}^{*}\right)^{-1}$

$\cdot \hat{\boldsymbol{G}}_{1}^{\mathrm{T}} \Delta \boldsymbol{g}_{1, k}^{*}+\Delta \boldsymbol{g}_{1, k}^{\mathrm{T}} \hat{\boldsymbol{G}}_{1}^{*}\left(\hat{\boldsymbol{G}}_{1}^{\mathrm{T}} \hat{\boldsymbol{G}}_{1}^{*}\right)^{-1} \boldsymbol{P}\left(\hat{\boldsymbol{G}}_{1}^{\mathrm{H}} \hat{\boldsymbol{G}}_{1}\right)^{-1} \hat{\boldsymbol{G}}_{1}^{\mathrm{H}} \Delta \boldsymbol{G}_{1}$

$\left.\cdot \boldsymbol{A} \Delta \boldsymbol{G}_{1}^{\mathrm{H}} \hat{\boldsymbol{G}}_{1}\left(\hat{\boldsymbol{G}}_{1}^{\mathrm{H}} \hat{\boldsymbol{G}}_{1}\right)^{-1} \boldsymbol{P}\left(\hat{\boldsymbol{G}}_{1}^{\mathrm{T}} \hat{\boldsymbol{G}}_{1}^{*}\right)^{-1} \hat{\boldsymbol{G}}_{1}^{\mathrm{T}} \Delta \boldsymbol{g}_{1, k}^{*}\right]$

$=a_{z f}^{2}\left[\sum_{i=1}^{2 K}\left(1+v_{i}\right) P_{s, i} \varepsilon_{1, i} \frac{\mathbf{1}_{k^{\prime}}\left(\boldsymbol{Q}_{1}\right)^{-1} \mathbf{1}_{k^{\prime}}^{\mathrm{T}}}{M}+\varepsilon_{1, k} \frac{\operatorname{Tr}\left[\left(\boldsymbol{Q}_{1}\right)^{-1} \boldsymbol{P} \boldsymbol{A P}\right]}{M}\right]$

$+\varepsilon_{1, k} \sum_{i=1}^{2 K}\left(1+v_{i}\right) P_{s, i} \varepsilon_{1, i} \frac{\operatorname{Tr}\left\{\left[\left(\boldsymbol{Q}_{1}\right)^{-1} \boldsymbol{P}\right]^{2}\right\}}{M^{2}}$

$=a_{z f}^{2}\left[\frac{\eta_{1, k^{\prime}}^{-1}}{M} \sum_{i=1}^{2 K}\left(1+v_{i}\right) P_{s, i} \varepsilon_{1, i}+\frac{\varepsilon_{1, k}}{M} \sum_{i=1}^{2 K}\left(1+v_{i}\right) P_{s, i} \eta_{1, i^{\prime}}^{-1}\right.$

$\left.+\frac{\varepsilon_{1, k}}{M^{2}} \sum_{i=1}^{2 K}\left(1+v_{i}\right) P_{s, i} \varepsilon_{1, i} \sum_{i=1}^{2 K} \eta_{1, i}^{-1} \eta_{1, i^{\prime}}^{-1}\right]$.

Compute E[|y $\left.\left.\left.\right|^{n}\right|^{2}\right]$ : According to Eqs.(5) and (19), $\mathrm{E}\left\{\boldsymbol{n}_{R} \boldsymbol{n}_{R}{ }^{\mathrm{H}}\right\}=\sigma \boldsymbol{I}_{M}$ and with property $\hat{\boldsymbol{G}}_{1}$ and $\Delta \boldsymbol{G}_{1}$ independent, we have

$$
\begin{aligned}
& \mathrm{E}\left[\left|y^{n}\right|^{2}\right]=a_{z f}^{2} \sigma \mathrm{E}\left[\mathbf{1}_{k^{\prime}}\left(\hat{\boldsymbol{G}}_{1}^{\mathrm{H}} \hat{\boldsymbol{G}}_{1}\right)^{-1} \mathbf{1}_{k^{\prime}}^{\mathrm{T}}\right. \\
& +\Delta \boldsymbol{g}_{1, k}^{\mathrm{T}} \hat{\boldsymbol{G}}_{1}^{*}\left(\hat{\boldsymbol{G}}_{1}^{\mathrm{T}} \hat{\boldsymbol{G}}_{1}^{*}\right)^{-1} \times \boldsymbol{P}\left(\hat{\boldsymbol{G}}_{1}^{\mathrm{H}} \hat{\boldsymbol{G}}_{1}\right)^{-1} \\
& \left.\hat{\boldsymbol{G}}_{1}^{\mathrm{H}} \hat{\boldsymbol{G}}_{1}\left(\hat{\boldsymbol{G}}_{1}^{\mathrm{H}} \hat{\boldsymbol{G}}_{1}\right)^{-1} \boldsymbol{P}\left(\hat{\boldsymbol{G}}_{1}^{\mathrm{T}} \hat{\boldsymbol{G}}_{1}^{*}\right)^{-1} \hat{\boldsymbol{G}}_{1}^{\mathrm{T}} \Delta \boldsymbol{g}_{1, k}^{*}\right] \\
& =a_{z f}^{2} \sigma\left[\frac{\mathbf{1}_{k^{\prime}}\left(\boldsymbol{Q}_{1}\right)^{-1} \mathbf{1}_{k^{\prime}}^{\mathrm{T}}}{M}+\varepsilon_{1, k} \frac{\left.\operatorname{Tr}\left[\left(\boldsymbol{Q}_{1}\right)^{-1} \boldsymbol{P}\left(\boldsymbol{Q}_{1}\right)^{-1} \boldsymbol{P}\right]\right\}}{M^{2}}\right] \\
& =a_{z f}^{2} \sigma\left[\frac{\eta_{1, k^{\prime}}^{-1}}{M}+\frac{\varepsilon_{1, k}}{M^{2}} \sum_{i=1}^{2 K} \eta_{1, i}^{-1} \eta_{1, i^{\prime}}^{-1}\right] .
\end{aligned}
$$

Compute E $\left[\left|y^{r}\right|^{2}\right]$ : According to Eq.(16), similarly to Eq.(21), we have

$$
\begin{aligned}
& \mathrm{E}\left[\left|y^{r}\right|^{2}\right] \\
& =a_{z f}^{2} P_{R}^{r}\left[\frac{\mathbf{1}_{k^{\prime}}\left(\boldsymbol{Q}_{1}\right)^{-1} \mathbf{1}_{k^{\prime}}^{\mathrm{T}}}{M}+\varepsilon_{1, k} \frac{\left.\operatorname{Tr}\left[\left(\boldsymbol{Q}_{1}\right)^{-1} \boldsymbol{P}\left(\boldsymbol{Q}_{1}\right)^{-1} \boldsymbol{P}\right]\right\}}{M^{2}}\right] \\
& =a_{z f}^{2} P_{R}^{r}\left[\frac{\eta_{1, k^{\prime}}^{-1}}{M}+\frac{\varepsilon_{1, k}}{M^{2}} \sum_{i=1}^{2 K} \eta_{1, i}^{-1} \eta_{1, i^{\prime}}^{-1}\right] .
\end{aligned}
$$

Compute $\mathrm{E}\left[\left|y^{i 2}\right|^{2}\right]$ : Since $\mathrm{E}\left\{t_{R} t_{R}{ }^{\mathrm{H}}\right\}=\frac{v_{R} P_{R}}{M} \boldsymbol{I}_{M}$, we can obtain

$$
\mathrm{E}\left[\left|y^{\prime 2}\right|^{2}\right]=\frac{v_{R} P_{R}}{M} \mathrm{E}\left[\boldsymbol{g}_{1, k}^{\mathrm{T}} \boldsymbol{g}_{1, k}^{*}\right]=v_{R} P_{R} \beta_{1, k}
$$

Compute $\mathrm{E}\left[r_{k} r_{k}^{\mathrm{H}}\right]$ : According to Eqs.(20) (23), we have

$$
\begin{aligned}
& \mathrm{E}\left[r_{k} r_{k}^{\mathrm{H}}\right]=\mu_{k} \mathrm{E}\left\{a_{z f}^{2} P_{s, k^{\prime}}+a_{z f}^{2} \mathbf{1}_{k^{\prime}}, \Lambda_{1} \mathbf{1}_{k^{\prime}}^{\mathrm{T}}+\left|y^{\prime 1}\right|^{2}+\right. \\
& \left.+\left|y^{n}\right|^{2}+\left|y^{r}\right|^{2}+\left|y^{\prime 2}\right|^{2}+\sigma_{n}\right\}
\end{aligned}
$$

By using Eqs.(20) (24), we obtain Eq.(9).

C) Proof of Lemma 3

\section{$\mathrm{E}\left\{r_{R} r_{R}^{\mathrm{H}}\right\}$}

$=\mu_{R} \operatorname{diag}\left(\mathrm{E}\left[y_{R} y_{R}^{\mathrm{H}}\right]\right)$

$$
\begin{aligned}
& =\mu_{R} \operatorname{diag}\left(\mathrm{E}\left[\boldsymbol{G}_{2} \boldsymbol{A} \boldsymbol{G}_{2}^{\mathrm{H}}+\boldsymbol{G}_{R R} \frac{P_{R}}{M}\left(1+v_{R}\right) \boldsymbol{G}_{R R}^{\mathrm{H}}+\sigma \boldsymbol{I}_{M}\right]\right) \\
& =\mu_{R}\left(\sum_{i=1}^{2 K}\left(1+v_{i}\right) P_{s, i} \beta_{2, i}+\sigma_{r r}^{2}\left(1+v_{R}\right) P_{R}+\sigma\right) \boldsymbol{I}_{M} \\
& =P_{R}^{r 1} \boldsymbol{I}_{M} .
\end{aligned}
$$

Using the same approach as the Proof of Lemma 2, we have

$$
a_{z f^{\prime}}^{2} \stackrel{\text { a.s. }}{\longrightarrow} P_{R}\left[\operatorname { T r } \left\{\frac{\left(\boldsymbol{Q}_{3}\right)^{-1} \boldsymbol{P} \boldsymbol{A P}}{M}+\left(\sigma+\sigma_{r r}^{2}\left(1+v_{R}\right) P_{R}\right.\right.\right.
$$

$\left.\left.+P_{R}^{r 1}+\sum_{j=1}^{2 K}\left(1+v_{j}\right) P_{s, j} \varepsilon_{2, j}\right)\left[\frac{\left[\left(\boldsymbol{Q}_{2}\right)^{-1} \boldsymbol{P}\left(\boldsymbol{Q}_{3}\right)^{-1} \boldsymbol{P}\right]^{2}}{M^{2}}\right\}\right]^{-1}$.

Since $\operatorname{Tr}\left\{\left(\boldsymbol{Q}_{3}\right)^{-1} \boldsymbol{P} \boldsymbol{A} \boldsymbol{P}\right\}=\sum_{i=1}^{2 K}\left(1+v_{i}\right) P_{s, i} \eta_{3, i}^{-1}, \operatorname{Tr}\left\{\left(\boldsymbol{Q}_{2}\right)^{-1}\right.$ $\left.\boldsymbol{P}\left(\boldsymbol{Q}_{3}\right)^{-1} \boldsymbol{P}\right\}=\sum_{i=1}^{2 K} \eta_{2, i}^{-1} \eta_{3, i^{-1}}^{-1}$ then Eq.(12) can be easily 
obtained.

\section{D) Proof of Theorem 2}

Compute $\mathrm{E}\left[\left|y^{L I}\right|^{2}\right]$ : Since $\tilde{x}_{R} \tilde{x}_{R}^{\mathrm{H}}=\frac{P_{R}}{M} \boldsymbol{I}_{M}, \mathrm{E}\left[t_{R} t_{R}{ }^{\mathrm{H}}\right]=$ $\frac{v_{R} P_{R}}{M} \boldsymbol{I}_{M}$, and the property $\boldsymbol{G}_{R R}, \Delta \boldsymbol{g}_{3, k}, \hat{\boldsymbol{G}}^{2}$ and $\hat{\boldsymbol{G}}^{3}$ are independent, we can obtain

$\mathrm{E}\left[\left|y^{L I}\right|^{2}\right]=a_{z f^{\prime}}^{2} \frac{P_{R}}{M}\left(1+v_{R}\right) \operatorname{Tr}\left\{\mathrm{E}\left[\boldsymbol{G}_{R R} \boldsymbol{G}_{R R}^{\mathrm{H}} \mathbf{1}_{k^{\prime}}\left(\hat{\boldsymbol{G}}_{2}^{\mathrm{H}} \hat{\boldsymbol{G}}_{2}\right)^{-1} \mathbf{1}_{k^{\prime}}^{\mathrm{T}}\right.\right.$

$\left.\left.+\boldsymbol{G}_{R R} \boldsymbol{G}_{R R}^{\mathrm{H}} \Delta \boldsymbol{g}_{3, k}^{\mathrm{T}} \hat{\boldsymbol{G}}_{3}^{*}\left(\hat{\boldsymbol{G}}_{3}^{\mathrm{T}} \hat{\boldsymbol{G}}_{3}^{*}\right)^{-1} \boldsymbol{P}\left(\hat{\boldsymbol{G}}_{2}^{\mathrm{H}} \hat{\boldsymbol{G}}_{2}\right)^{-1} \boldsymbol{P}\left(\hat{\boldsymbol{G}}_{3}^{\mathrm{T}} \hat{\boldsymbol{G}}_{3}^{*}\right)^{-1} \hat{\boldsymbol{G}}_{3}^{\mathrm{T}} \Delta \boldsymbol{g}_{3, k}^{*}\right]\right\}$

$=a_{z f^{\prime}}^{2} \frac{P_{R}}{M}\left(1+v_{R}\right) \sigma_{r r}^{2}\left[\mathbf{1}_{k^{\prime}}\left(\boldsymbol{Q}_{2}\right)^{-1} \mathbf{1}_{k^{\prime}}^{\mathrm{T}}+\varepsilon_{3, k} \frac{\left.\operatorname{Tr}\left[\left(\boldsymbol{Q}_{2}\right)^{-1} \boldsymbol{P}\left(\boldsymbol{Q}_{3}\right)^{-1} \boldsymbol{P}\right]\right\}}{M^{2}}\right]$

$=a_{z f^{\prime}}^{2} \sigma_{r r}^{2}\left(1+v_{R}\right) P_{R}\left[\frac{\eta_{2, k^{\prime}}^{-1}}{M}+\frac{\varepsilon_{3, k}}{M^{2}} \sum_{i=1}^{2 K} \eta_{2, i}^{-1} \eta_{3, i^{\prime}}^{-1}\right]$.

The derivation of $\mathrm{E}\left[\left|y^{t 1}\right|^{2}\right], \mathrm{E}\left[\left|y^{n}\right|^{2}\right], \mathrm{E}\left[\left|y^{r}\right|^{2}\right], \mathrm{E}\left[\left.y^{t 2}\right|^{2}\right]$ and $\mathrm{E}\left[r_{k} r_{k}^{\mathrm{H}}\right]$ is similar to that of Theorem 1, thus, it is omitted.

\section{References}

[1] LARSSON E G, TUFVESSON F, EDFORS O, et al. Massive MIMO for next generation wireless systems[J]. IEEE commun. mag., 2014, 52(2): 186-195.

[2] KIM S J, DEVROYE N, MITRAN P, et al. Achievable rate regions and performance comparison of half duplex protocols[J]. IEEE trans. inf. theory, 2011, 57(10): 6405-6418.

[3] RODRIGUEZ L J, TRAN N, NGOC T L. Optimal power allocation and capacity of full-duplex AF relaying under residual selfinterference[J]. IEEE wireless commun. lett., 2014, 3(2): 233-236.

[4] XIA X C, XU K, ZHANG D M, et al. Low-complexity transceiver design and antenna subset selection for soop-erative half and full-duplex relaying systems[C]//IEEE Global Communications
Conference, Austin, USA, 2014: 3314-3319.

[5] YANG J, FAN P, DUONG T Q, et al. Exact performance of two-way AF relaying over Nakagami-m fading environment[J]. IEEE trans. wireless commun., 2011, 10(3): 980-987.

[6] CUI H, SONG L, JIAO B. Multi-pair two-way amplify-and-forward relaying with very large number of relay antennas[J]. IEEE Trans. Wireless Commun., 2014, 13(5): 2636-2645.

[7] SURAWEERA H A. Multi-pair amplify-and-forward relaying with very large antenna arrays $[\mathrm{C}] /$ Proceeding IEEE ICC, Budapest, Hungary, 2013: 4635-4640.

[8] XIA X C, XIE W, ZHANG D M, et al. Multi-pair fullduplex amplifyand-forward relaying with very large antenna arrays[C]// IEEE Wireless Communications and NETWORKING Conference, New Orleans, USA, 2015: m304-309.

[9] SUN X L, XU K, MA W F, et al. Multi-pair two-way massive MIMO AF full-duplex relaying with imperfect CSI over ricean fading channels[J]. IEEE access, 2016, 4: 4933-4945.

[10] BJORNSON E, HOYDIS J, KOUNTOURIS M, et al. Massive MIMO systems with non-ideal hardware: energy efficiency, estimation, and capacity limits[J]. IEEE trans. inf. theory, 2014, 60(11): 7112-7139

[11] GUSTAVSSON U. On the impact of hardware impairments on massive MIMO[C]//IEEE GLOBECOM Workshop, Austin, USA, 2014: 294-300.

[12] XIA X C, ZHANG D M, XU K, et al. Hardware impairments aware transceiver for full-duplex massive MIMO relaying[J]. IEEE trans. signal processing, 2015, 63(24): 6565-6580.

[13] ZHANG Z Z, CHEN Z, SHEN M, et al. Spectral and energy efficiency of multipair two-way full-duplex relay systems with massive MIMO[J]. IEEE journal on selected areas in communications, 2016, 34(4): 848-863.

[14] ZHANG Q, JIN S, WONG K K, et al. Power scaling of up-link massive MIMO system with arbitrary-rank channel means[J]. IEEE $\mathrm{j}$. sel. signal process., 2014,8 (5): 966-981.

\section{About the authors}

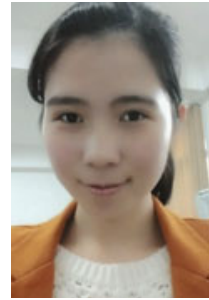

SUN Xiaoli was born in 1992. She received her B.E. from PLAUST (PLA University of Science and Technology), Nanjing, China, in 2014. She is currently working toward the B.S. degree in Institution of Communications Engineering, PLAUST. Her research interests include MIMO techniques, relaying network, full-duplex communication, Network coding.

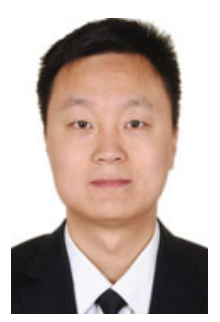

XU Kui [corresponding author] was born in 1982. He received the B.S. degree in wireless communications from the PLAUST (PLA University of Science and Technology), Nanjing, China in 2004, and the Ph.D. degree in software defined radio from PLAUST, in 2009. He is currently an associate professor in the College of Communications Engineering, PLAUST. 
From Dec. 2013, he was a Postdoctoral Fellow with PLAUST. His research interests include broadband wireless communications, signal processing for communications, network coding, wireless communication networks. He is the author of about 100 papers in refereed journals and conference proceedings and holds 5 patents in China.

He currently served on the technical program committee of the IEEE WCSP 2014, WCSP 2015, ICSPDM 2015, SIRS 2015 TPC. He received the URSI Young Scientists Award in 2014 and the 2010 ten excellent doctor degree dissertation award of PLAUST. He serves as the reviewer for IEEE Trans. Wireless Communication, IEEE Transaction Vehicle Technology, IEEE Communications Letter, IEEE Signal Processing Letters. (Email: lgdxxukui@126.com)

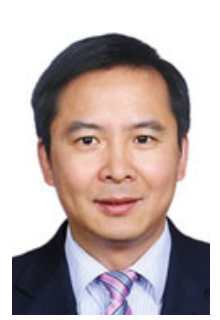

XU Youyun was born in 1966. He graduated from Shanghai Jiao Tong University with a Ph.D. degree in information and communication engineering in 1999.

$\mathrm{He}$ is currently a professor with the Nanjing Institute of Communication Engineering, China. $\mathrm{He}$ is also a part-time professor with the Institute of Wireless Communication Technology of Shanghai Jiao Tong University (SJTU), Shanghai, China. He has more than 20 years professional experience of teaching and researching in communication theory and engineering. Now, his research interests are focusing on new generation wireless mobile communication systems (IMT-advanced and related), advanced channel coding and modulation techniques, multi-user information theory and radio resource management, wireless sensor networks, cognitive radio networks, etc. $\mathrm{He}$ is a senior member of IEEE, and a senior member of the Chinese Institute of Electronics.

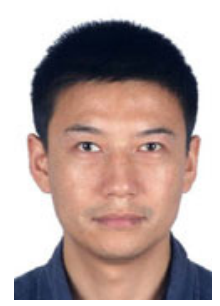

MA Wenfeng was born in 1974. He received the B.S. degree in microwave communication from the Institute of Communications Engineering, Nanjing, China, in 1995, and the M.S. degree in communications and information system from the Institute of Communications Engineering, Nanjing, China, in 1998, and the Ph.D. degree in communications and information system from the PLAUST (PLA University of Science and Technology), Nanjing, China, in 2002. From Apr. 2004 to Feb. 2006, he was a Postdoctoral Fellow with Shanghai Jiao Tong University (SJTU), Shanghai, China.

$\mathrm{He}$ is currently a associate professor with the PLAUST. His research interests include wireless communication networks, broadband wireless communications. 